Louisiana State University

LSU Digital Commons

3-1-2004

\title{
NMR difference spectroscopy with a dual saddle-coil difference probe
}

Megan A. MacNaughtan

Purdue University

Aaron P. Smith

Purdue University

Peter B. Goldsbrough

Purdue University

Robert E. Santini

Purdue University

Daniel Raftery

Purdue University

Follow this and additional works at: https://digitalcommons.Isu.edu/chemistry_pubs

\section{Recommended Citation}

MacNaughtan, M., Smith, A., Goldsbrough, P., Santini, R., \& Raftery, D. (2004). NMR difference

spectroscopy with a dual saddle-coil difference probe. Analytical and Bioanalytical Chemistry, 378 (6), 1520-1527. https://doi.org/10.1007/s00216-003-2374-3

This Article is brought to you for free and open access by the Department of Chemistry at LSU Digital Commons. It has been accepted for inclusion in Faculty Publications by an authorized administrator of LSU Digital Commons.

For more information, please contact ir@lsu.edu. 
Louisiana State University

LSU Digital Commons

Faculty Publications

Department of Biological Sciences

3-1-2004

\section{NMR difference spectroscopy with a dual saddle-coil difference probe}

Megan A. MacNaughtan

Purdue University

Aaron P. Smith

Purdue University

Peter B. Goldsbrough

Purdue University

Robert E. Santini

Purdue University

Daniel Raftery

Purdue University

Follow this and additional works at: https://digitalcommons.Isu.edu/biosci_pubs

\section{Recommended Citation}

MacNaughtan, M., Smith, A., Goldsbrough, P., Santini, R., \& Raftery, D. (2004). NMR difference

spectroscopy with a dual saddle-coil difference probe. Analytical and Bioanalytical Chemistry, 378 (6), 1520-1527. https://doi.org/10.1007/s00216-003-2374-3

This Article is brought to you for free and open access by the Department of Biological Sciences at LSU Digital Commons. It has been accepted for inclusion in Faculty Publications by an authorized administrator of LSU Digital Commons. For more information, please contact ir@lsu.edu. 


\section{Megan A. Macnaughtan · Aaron P. Smith \\ Peter B. Goldsbrough · Robert E. Santini \\ Daniel Raftery \\ NMR difference spectroscopy with a dual saddle-coil difference probe}

Received: 18 August 2003 / Revised: 21 October 2003 / Accepted: 27 October 2003 / Published online: 10 January 2004

(C) Springer-Verlag 2004

\begin{abstract}
A new difference probe for nuclear magnetic resonance (NMR) spectroscopy is presented. The difference probe uses two saddle-shaped coils to excite and detect two samples simultaneously. The samples are held in a specially modified 3-mm NMR tube with an Ultem plastic disk to separate the samples. The probe's resonant circuit contains two crossed diodes that passively switch the relative phase of each coil during the NMR experiment. The result is a difference spectrum from the two samples. The degree of cancellation of common signals was determined to be approximately $90 \%$, and the application of the probe to relaxation-edited difference spectroscopy for identifying protein-ligand interactions was demonstrated using glutathione and glutathione $S$-transferase binding protein.
\end{abstract}

Keywords Difference spectroscopy $\cdot$ Background subtraction · Saddle coils · Protein-ligand interactions . Relaxation

\section{Introduction}

Comparing two sets of data is a fundamental aspect of analytical chemistry. Once an analytical technique is established, its usefulness is tested for distinguishing differences between samples, whether those differences are concentration, chemical, or physical. While most differences are observed through individual analysis of two spectra, subtraction techniques can often reveal subtle differences and eliminate interfering background signals. Subtrac-

M. A. Macnaughtan · R. E. Santini · D. Raftery $(\bowtie)$

Department of Chemistry, Purdue University,

West Lafayette, IN 47907, USA

Tel.: +1-765-494-6070, Fax: +1-765-496-1200,

e-mail: raftery@purdue.edu

A. P. Smith · P. B. Goldsbrough

Department of Horticulture and Landscape Architecture,

Purdue University, West Lafayette, IN 47907, USA tions can be performed either using the hardware of the spectrometer or with software techniques. When two samples are analyzed individually in a serial fashion, often the spectra are postprocessed with subtraction software. Alternatively, subtraction can be implemented through the hardware by analyzing two samples simultaneously with similar or identical sources and detectors. Several light spectroscopies such as ultraviolet, visible, infrared, atomic absorption, and fluorescence spectroscopy use double-beam instruments to achieve hardware-based subtraction [1]. In nuclear magnetic resonance (NMR) spectroscopy, difference spectroscopy obtained from two samples utilizing the probe hardware was only recently demonstrated by our group using a solenoidal microcoil difference probe [2]. The difference probe uses one receiver, but has two coils, one to excite and detect the NMR signal from each sample. The transmitter source and receiver are not modified to create the subtraction; the difference is a result of switching the resonant circuit so that the coils generate opposite phases with respect to each other during data acquisition.

NMR is a particularly useful analytical technique because a wide variety of differences can be exploited to reveal information about an individual analyte, a mixture, or even a dynamic system. The most basic and commonly used comparisons in NMR are differences in chemical shifts or coupling constants, which can be used to identify an analyte and determine its chemical structure. For example, differences in the chemical shifts in ${ }^{1} \mathrm{H}$ and ${ }^{15} \mathrm{~N}-\mathrm{HSQC}$ spectra are used to screen for potential drug candidates by observing changes in the target protein spectrum in the presence and absence of a binding ligand $[3,4]$. Protein-ligand interactions are commonly investigated with NMR difference techniques because binding ligands can be identified through changes in the ligand's translational diffusion rate, relaxation rate, or chemical shift upon interaction with the target protein [5]. These difference methods either use two samples (a ligand-only sample and a ligand-protein mixture) with the same NMR experiment or use one ligand-protein sample and perform two different experiments by changing the NMR pulse sequence. Two magnetization transfer techniques, reverse NOE pumping 
[6] and saturation-transfer difference (STD) spectroscopy [7] alter the pulse sequence to obtain two spectra on the same sample. The spectra can be subtracted in software or a difference spectrum can be produced directly by altering the pulse sequence with phase cycling.

While these techniques produce difference spectra efficiently using a single sample, other techniques for determining binding ligands, such as relaxation- and diffusionedited NMR [8], require two samples. Transverse relaxation and diffusion are two physical properties of ligands that change when the ligand binds to a protein. Bound ligands exhibit faster transverse relaxation rates and slower diffusion rates compared to the free ligands. In relaxationedited NMR, the Carr-Purcell Meiboom-Gill (CPMG) pulse sequence $[9,10]$ is used to attenuate the NMR signals based on the transverse relaxation rate. A reference sample of ligands in the presence of protein is subtracted from a sample consisting of ligands only with their original, slower relaxation rate. The difference spectrum reveals the signal of the binding ligands alone.

In this article we present a new method for obtaining an NMR difference spectrum of two samples simultaneously without phase cycling, experimental changes, or additional software. A dual saddle-coil NMR difference probe was built using the "double-beam" approach to difference spectroscopy. Like our solenoidal microcoil difference probe [2], crossed diodes are used to switch the circuit pathway passively, and consequently, the relative phase of the coils during the NMR experiment. Only one free induction decay is collected and Fourier transformed to give a difference spectrum consisting of signals from both samples that are $180^{\circ}$ out-of-phase with respect to each other. The saddle-coil difference probe has two saddleshaped Helmholtz coils that excite and detect two samples placed in a $3 \mathrm{~mm}$, vertically aligned NMR tube. Compared to the microcoil difference probe, the saddle-coil probe has some additional features to improve the performance of NMR difference experiments on protein-ligand systems. These enhancements include a larger sample detection volume, lower limit of detection, sample-spinning capabilities, and an axial pulsed field gradient. To demonstrate the capabilities of the difference probe, a relaxationedited difference experiment was performed to examine interactions between glutathione or serine with the glutathione $S$-transferase, AtGSTU19, an enzyme known to bind glutathione [11].

\section{Experimental}

Difference circuit

The most important feature of the difference probe is the resonant circuit, where the difference between the samples' signals is generated. Unlike in a typical resonant circuit with one excitation/detection coil, the two excitation/detection coils in the difference circuit generate signals simultaneously. In addition, the circuit changes so that the coils are excited and detected with different circuit pathways. Crossed diodes are used to switch the circuit passively during the NMR experiment. During excitation, the pulse used to excite the samples through the saddle coils also activates the crossed

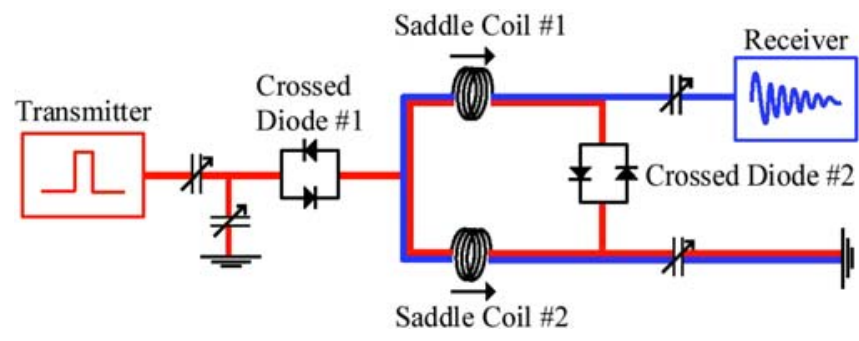

Fig. 1 The difference circuit includes four variable capacitors, two excitation/detection saddle coils, two crossed diodes, the transmitter where the excitation pulses are generated, and the receiver where the NMR signal is collected. The red lines indicate the circuit path during excitation when the crossed diodes are activated The blue lines indicate the circuit path during detection when the crossed diodes are not activated. Notice that the relative orientation of the saddle coils with respect to the transmitter during excitation is parallel, and to the receiver during detection is serial, producing a $180^{\circ}$ phase difference between the signals received from each coil. This change in orientation causes common magnetization, indicated by the black arrows near the coils, to cancel upon summation along the blue path

diodes so that they allow current to flow. The result is a circuit pathway in which the saddle coils are connected in parallel with respect to the transmitter (see Fig. 1, red pathway). However, during detection, the NMR signal voltage produced by the samples is small enough that the crossed diodes are not active and do not allow current to flow. The circuit pathway changes such that the saddle coils are connected in series with respect to the receiver as shown in Fig. 1 along the blue pathway. The parallel excitation and serial detection creates a phase difference of $180^{\circ}$ between the signals from each coil. The result is the generation of an automatic difference between the signals through the circuit hardware. The difference signals are collected into one free induction decay that is digitally stored and Fourier transformed to give a difference spectrum of the two samples.

\section{Reagents}

Methanol, acetonitrile (Mallinckrodt Laboratory Chemicals, Phillipsburg, NJ, USA), ethanol (Pharmco Products, Inc., Brookfield, CT, USA), and $\mathrm{D}_{2} \mathrm{O}(99.9 \% \mathrm{D}$, Cambridge Isotope Laboratories, Inc. Andover, MA, USA) were used to make standard samples for testing the difference probe. The relaxation-edited difference experiments were performed using recombinant glutathione $S$-transferase AtGSTU19 (GST) [11], glutathione (Sigma-Aldrich Co., Milwaukee, WI, USA), and serine (Sigma-Aldrich Co., Milwaukee, WI, USA). Glutathione $S$-transferases are a family of multifunctional enzymes present in virtually all organisms whose primary activity is to catalyze the conjugation of glutathione (GSH, $\gamma$-L-glutamyl-Lcysteinyl-L-glycine) to a number of substrates and therefore, exhibit binding to GSH [12]. The GST was expressed in E. coli and purified via glutathione-affinity chromatography as previously described [11]. The GST was washed and concentrated in $\mathrm{D}_{2} \mathrm{O}$ using an Amicon ultra-4 centrifugal filter unit (Millipore Corp., Billerica, MA, USA).

\section{Difference probe}

The dual-saddle NMR difference probe was built for a $300 \mathrm{MHz}$, wide-bore $(73 \mathrm{~mm})$ magnet. The top of the probe contains the difference circuit with the saddle coils, the pulse field gradient coils, and the hardware to support the coils and allow for sample spinning (see Fig. 2). The difference circuit is connected to the transmitter and receiver of the spectrometer through coaxial cables and $\mathrm{BNC}$ connectors at the base of the probe. The actively shielded, 


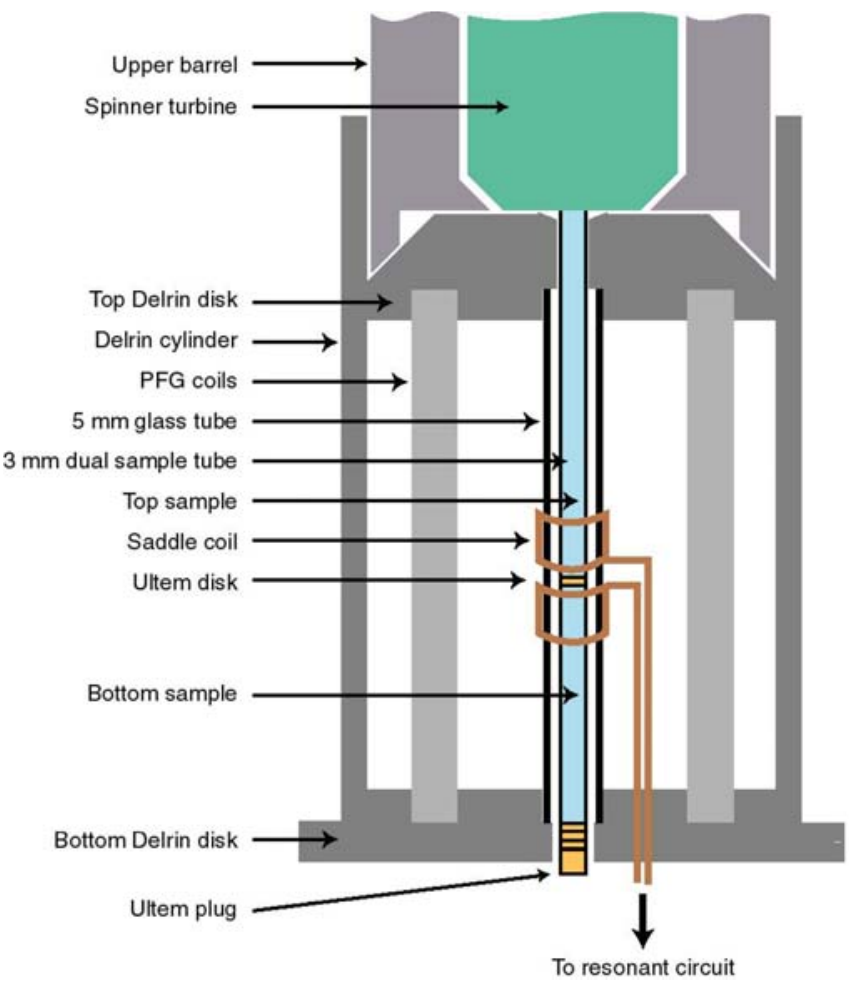

Fig. 2 The diagram of the saddle-coil difference probe head shows the orientation of the saddle coils and the dual-sample tube. The upper barrel fits inside the top of the difference probe so that the sample tube can be inserted into the difference probe using a spinner turbine. The $3 \mathrm{~mm}$ dual-sample tube fits inside the $5 \mathrm{~mm}$ coil support tube with the thin Ultem disk placed such that it sits between the saddle coils. The top and bottom Delrin disks and Delrin cylinder hold the support tube, saddle coils, and the pulsed field gradient (PFG) coils in place and allow for alignment with the upper barrel

pulsed field gradient coils were made in-house and consisted of two oppositely wound coils each supported by a cylinder made of Delrin plastic. The gradient coil design and construction has been described previously [13]. The saddle coils were constructed from $0.534 \mathrm{~mm}$ diameter, zero magnetic susceptibility $\mathrm{Cu}-\mathrm{Al} \mathrm{NMR}$ coil wire (Doty Scientific, Inc., Columbia, SC, USA). Aluminum forms were used to make the basic, flat Helmholtz coil shape with $5 \times$ $5 \mathrm{~mm}$ squares, and then the coils were hand-formed into the saddle coil shape around a 5-mm glass tube (see Fig. 3). As shown in Fig. 2, the 5-mm glass support tube was held in place inside the top of the probe by two disks and a connecting cylinder made from Delrin plastic. The coils were rigid enough that no glue was needed to hold the coils against the 5-mm glass tube; however, to prevent the coils from sliding down the glass tube, the coil leads were drawn through a hole in the bottom Delrin disk and glued in place with 5-minute epoxy (Devcon, Danvers, MA, USA). The rest of the difference circuit, including four variable capacitors (Voltronics, Denville, NJ, USA) for tuning and matching and the two nonmagnetic crossed diodes, were placed immediately underneath the bottom Delrin disk. Details regarding the difference circuit were described previously for the solenoidal microcoil difference probe developed in our laboratory [2].

The difference probe was designed to allow a 3-mm NMR sample tube to be inserted into the probe using the traditional, airsupported drop from the top of the magnet through the upper barrel with a spinner turbine. The top Delrin disk of the difference probe has a $4 \mathrm{~mm}$ hole through the concentric center and holds the $5 \mathrm{~mm}$ support tube in place to receive the sample. The upper barrel sits on the top Delrin disk inside the Delrin cylinder of the differ-

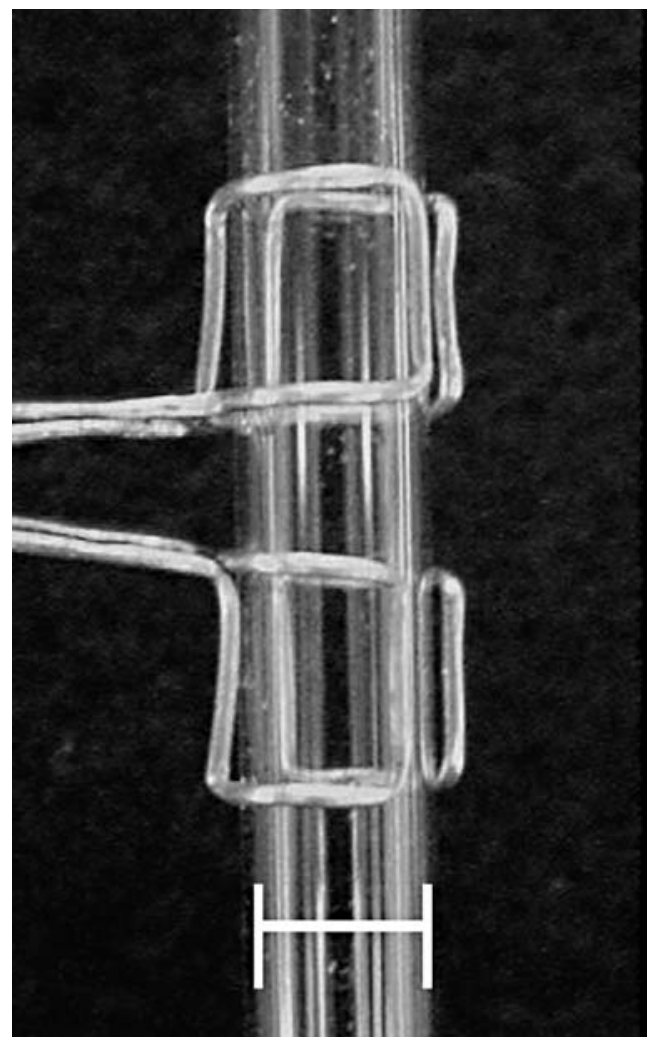

Fig. 3 Photograph of the saddle coils from the difference probe. The coils are supported on a $5-\mathrm{mm}$ glass tube marked by the white lines

ence probe (see Fig. 2). The 3-mm NMR tube is inserted into the $5 \mathrm{~mm}$ support tube and can be spun at $20 \mathrm{~Hz}$ using the normal software options.

The difference probe is designed to analyze two samples simultaneously, so a $3 \mathrm{~mm}$ NMR tube (Wilmad-Labglass, Buena, NJ, USA) was modified to hold two samples. The sealed end of the tube was cut and a small cylinder ( $3 \mathrm{~mm}$ long) of Ultem plastic was positioned inside the bottom of the tube as shown in Figs. $4 \mathrm{a}$ and $4 \mathrm{c}$. The cylinder was threaded and a matching Ultem screw was machined to provide a liquid-tight seal. A $0.5-\mathrm{mm}$ disk of Ultem shown in Figs. $4 \mathrm{a}$ and $4 \mathrm{~b}$ was inserted inside the $3 \mathrm{~mm}$ NMR tube at $61 \mathrm{~mm}$ from the bottom of the tube and was used to isolate the samples. All of the Ultem parts were made in the Jonathan Amy Facility for Chemical Instrumentation at Purdue University using Doty magnetic susceptibility matching plugs (Wilmad-Labglass, Buena, NJ, USA). The bottom sample was loaded into the tube first by inverting the tube. After the tube was sealed with the Ultem screw, the top sample was loaded. An unmodified $3 \mathrm{~mm}$ Varian spinner turbine was used to insert the samples into the difference probe and to spin the samples. The Ultem disk that separated the samples was positioned to sit between the two saddle coils of the difference probe (see Fig. 2).

\section{NMR spectroscopy}

All spectra were acquired on a Varian INOVA spectrometer operating at $300 \mathrm{MHz}$ for ${ }^{1} \mathrm{H}$. Initial shimming of the saddle coils was performed with a common, two-capacitor resonant circuit with the coils connected in parallel during excitation and detection. Gradient shimming was used for the axial shims. Subsequent shimming, with the saddle coils connected to the difference circuit, was performed manually. Each spectrum was processed with $1 \mathrm{~Hz}$ line broadening (unless otherwise stated) and zero-filled twice. 


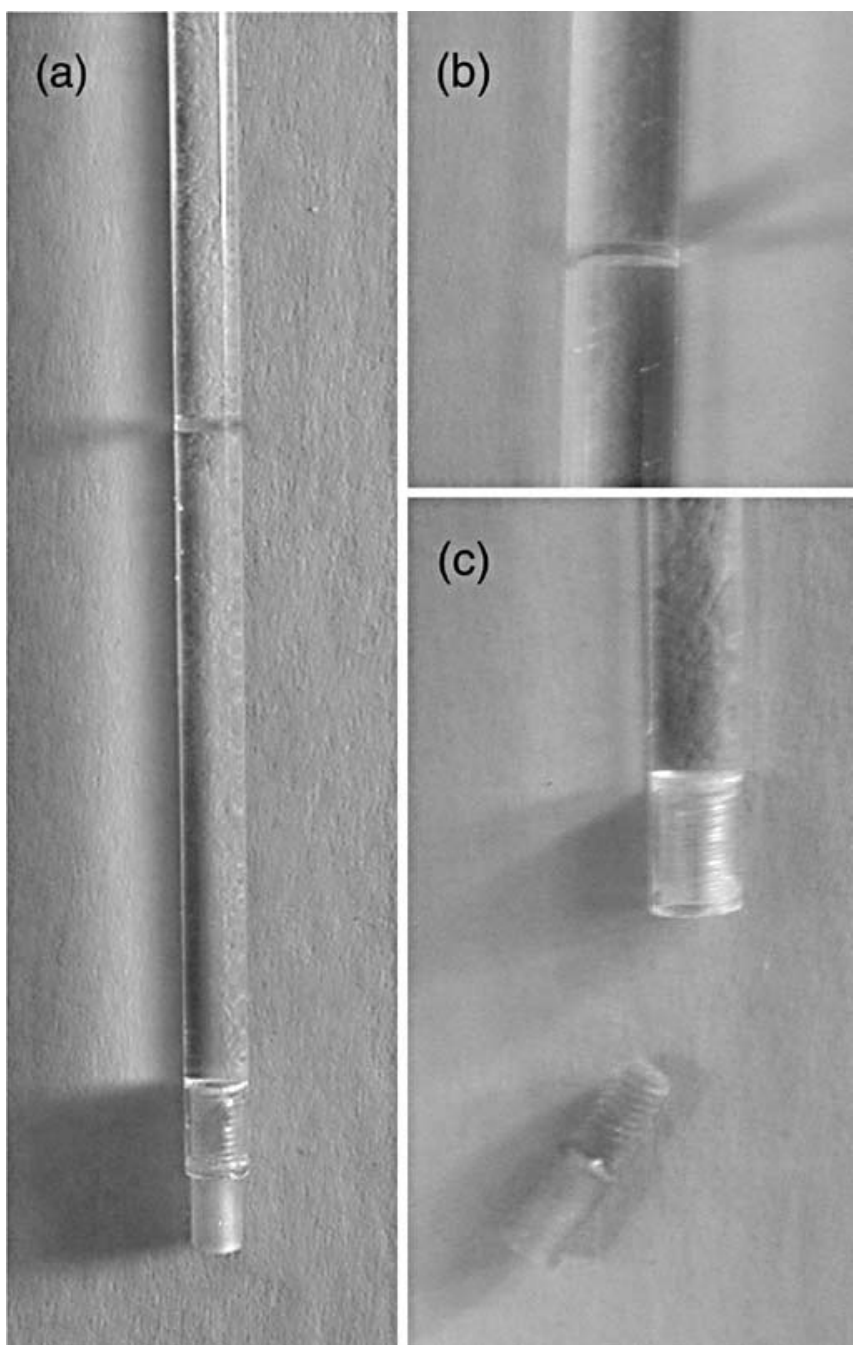

Fig. 4a-c Photographs of the modified 3-mm NMR tube used to hold two samples. a The dual sample tube showing the $0.5 \mathrm{~mm}$ Ultem disk, which separates the two samples, and the threaded cylinder and screw used to seal the bottom sample. b Enlarged view of the disk and $\mathbf{c}$ the threaded cylinder at the bottom of the tube with the screw disconnected

Solutions of methanol $(1 \mathrm{M})$ /acetonitrile $(1 \mathrm{M})$ and ethanol $(1 \mathrm{M})$ / acetonitrile $(1 \mathrm{M})$ in $\mathrm{D}_{2} \mathrm{O}$ were used to optimize the difference spectrum. Since acetonitrile was common to both samples, it was used to match the magnetic field strength and, thus, the chemical shift axis, of each sample and to determine the degree of cancellation. Since the acetonitrile and methanol were present at the same concentration, the cancellation factor was calculated by dividing the signal-to-noise ratio $(\mathrm{S} / \mathrm{N})$ of the methanol signal by the $\mathrm{S} / \mathrm{N}$ of the residual signal from the overlapped and subtracted acetonitrile peaks. The methanol and ethanol peaks were used to calibrate the pulse times and to monitor the peak shape and linewidth produced by each coil during shimming and optimization of the cancellation factor.

The CPMG-based relaxation-edited difference experiment was performed using a sample of GSH $(10 \mathrm{mM})$ and serine $(10 \mathrm{mM})$ and a sample of GSH $(10 \mathrm{mM})$ and serine $(10 \mathrm{mM})$ with $6.25 \mathrm{~g} / \mathrm{L}$ GST $[8,9,10]$. Both samples were prepared in a $0.2 \mathrm{M} \mathrm{PO}_{4}$ buffer (pH 6.6). A CPMG pulse sequence was used $\left(\mathrm{d} 1-90_{x}-\left(\tau-180_{y}-\tau\right)_{n^{-}}\right.$ acquire) with composite pulses, $\mathrm{d} 1=2 \mathrm{~s}$ (equilibrium delay), $\tau=$ $4 \mathrm{~ms}, n=50$ for a total spin-lock time of $400 \mathrm{~ms}$, an acquisition time of $1.5 \mathrm{~s}$, and 512 scans. The composite $90_{x}$ pulse consisted of $90_{y}-90_{-x}-90_{-y}-90_{x}$ and the $180_{y}$ pulse of $90_{x}-180_{y}-90_{x}$ [14].

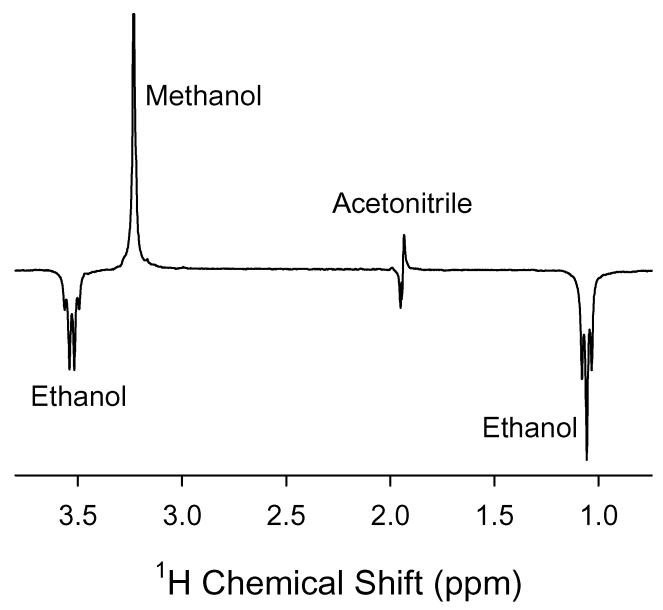

Fig. 5 Difference spectrum taken with the saddle-coil difference probe using $1 \mathrm{M}$ methanol/1 $\mathrm{M}$ acetonitrile as the top sample and $1 \mathrm{M}$ ethanol/1 $\mathrm{M}$ acetonitrile as the bottom sample. The acetonitrile peaks $(1.94 \mathrm{ppm})$ are overlapped and cancelled to demonstrate the automatic subtraction of signals with the difference probe. The methanol and ethanol peaks are used to monitor the individual response from each coil. Notice the $180^{\circ}$ phase difference between the methanol and ethanol signals

\section{Results and discussion}

For diagnostic purposes, the dual-sample $3 \mathrm{~mm}$ tube was loaded with two samples: $1 \mathrm{M}$ methanol and $1 \mathrm{M}$ acetonitrile in the top compartment and $1 \mathrm{M}$ ethanol and $1 \mathrm{M}$ acetonitrile in the bottom compartment. The difference spectrum obtained with these samples is shown in Fig. 5 using a single $90^{\circ}$ pulse-acquire pulse sequence. The methanol and ethanol peaks are clearly visible and show the $180^{\circ}$ phase difference generated by the unique resonant circuit of the difference probe. The reduced intensity of the acetonitrile signal at $1.94 \mathrm{ppm}$ demonstrates the subtraction that takes place in the circuit of the probe during acquisition. Since both samples have the same concentration of acetonitrile, the signals produced by each sample destructively interfere and cancel. The remaining signal or residual indicates the quality of the difference spectrum. Ideally, the residual acetonitrile signal should be zero, but due to slight differences in the geometry of the saddle coils and even the sample concentrations, a residual signal survives, producing a finite cancellation factor. The cancellation factor for the residual signal in Fig. 5 is 8.6, given by the ratio of the methanol and acetonitrile peaks.

The most important factors for optimizing the difference spectrum and improving the cancellation factor are matching the lineshape and frequency axes of the two coils. Both factors involve shimming to improve the homogeneity of the static magnetic field around the two samples and coils in the probe. As is well known in NMR, a homogeneous field will produce a narrow NMR line, thus improving resolution and sensitivity. Therefore, a narrow line is favorable, but with two coils inside one NMR probe, there are other factors for which the shims have to compensate. Namely, matching the magnetic field 


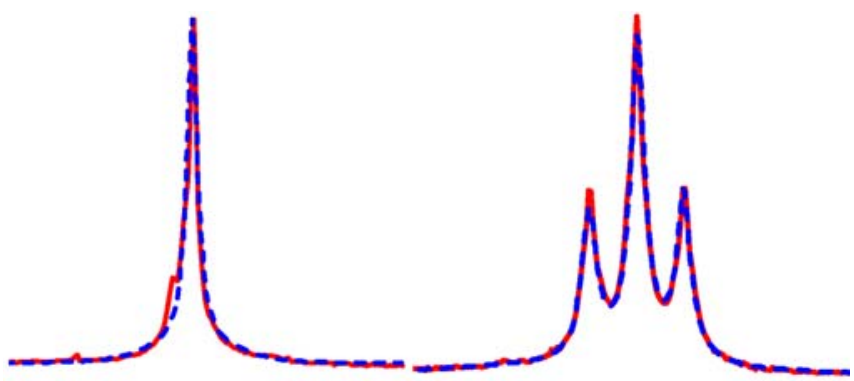

Fig. 6 The methanol and ethanol peaks from the difference spectrum in Fig. 5 were processed without line broadening (red lines) and fit with a combination of Lorentzian and Gaussian lineshapes with variable peak height, linewidth, fraction of Lorentzian and Gaussian lineshape, and peak frequency (blue lines). The phase of the ethanol peak was flipped and the vertical scales were normalized for illustration purposes. Both the methanol peak and the center peak of the ethanol triplet were found to have high Lorentzian character with $95 \%$ for methanol and $94 \%$ for ethanol. The halfheight Lorentzian linewidth for methanol was $2.13 \mathrm{~Hz}$ and the center line of ethanol was $2.43 \mathrm{~Hz}$

strength or frequency axis of each coil and obtaining identical, narrow lineshapes for both samples simultaneously. The frequency axes of each coil are matched using the $\mathrm{z} 1$ shim, which produces a compensating linear gradient across the coils. The lineshape produced by each coil is improved and matched using the other shims. While each coil can currently be shimmed individually to a linewidth at half-height of $0.25 \mathrm{~Hz}$, the individual optimum set of shim values must be compromised to shim both coils simultaneously. The linewidth of the peaks in Fig. 5 without line broadening were simulated with a combination of Lorentzian and Gaussian lineshapes to determine the linewidth at half-height and the peak shape under the compromised shim set. The methanol peak has a half-height linewidth of $2.13 \mathrm{~Hz}$ and is $95 \%$ Lorentzian with a small downfield shoulder. The ethanol triplet center line has a half-height linewidth of $2.43 \mathrm{~Hz}$ with $94 \%$ Lorentzian character. Fig. 6 shows the actual (red line) and simulated (blue line) lineshapes of each coil.

Since shimming the two samples in the difference probe requires a compromise between the optimal values for each of the coils, other methods for improving the cancellation factor were explored. The coils themselves are hand formed and imperfect in shape. Coils that are identical with a clean saddle shape would improve the lineshape

Table 1 The difference spectra taken with the saddle-coil difference probe and the solenoidal microcoil difference probe reported previously [2] were analyzed to determine the effect of weighting and symmetry with respect to the shims. Zero magnetic susceptibility wire and $\mathrm{D}_{2} \mathrm{O}$ susceptibility matched Ultem plastic were used to avoid magnetic susceptibility transitions around the coil and samples, which would otherwise distort the magnetic field. The wire is composed of a copper shell surrounding an inner aluminum core. The copper is not coated and is subject to oxidation, which alters its zero susceptibility value. Commercial NMR coil manufacturers use special alloys to control the susceptibility of the NMR coil [15], and such advanced methods would likely improve the performance of the difference probe.

Weighting functions applied to the free induction decay can also be used to improve the cancellation factor of the probe, but the degree of improvement depends on the original peak shape. With our solenoidal microcoil difference probe [2], it was found that weighting functions could improve the cancellation factor almost two-fold while with the saddle difference probe, little improvement was observed (see Table 1). The lineshape produced by the solenoidal microcoil difference probe was $>95 \%$ Gaussian while the saddle-coil difference probe is $~ 94 \%$ Lorentzian. In addition, the cancellation for the solenoidal microcoils was better with a cancellation factor of 27 compared to the saddle coils' factor of 7.2 without the use of weighting functions. The $\mathrm{S} / \mathrm{N}$ ratio of the residual cancellation signal from the solenoidal microcoil difference probe did not change significantly with the application of the weighting functions, indicating that the cancellation signal was attenuated by the same degree as the noise in the spectrum. However, the residual cancellation signal from the saddlecoil difference probe was improved by the weighting functions almost as much as the methanol signal. Essentially, the residual signal was treated as a real signal resulting in only minimal improvement to the cancellation factor.

The fact that the cancellation and weighting function results are better for a Gaussian lineshape can best be explained by inspecting the Lorentzian and Gaussian lineshapes. The artifacts from the cancellation come from the edges of the lineshape as the center apexes are overlapped to reach a maximum degree of cancellation. For peaks with the same linewidth at half-height, the Gaussian lineshape drops to the baseline faster than the Lorentzian peak. A larger portion of the Lorentzian peak area is stored in the tails, which must be matched in addition to the center apex of the peaks for good cancellation; thus, the cancel-

functions on the residual acetonitrile cancellation $\mathrm{S} / \mathrm{N}$, methanol $\mathrm{S} / \mathrm{N}$, and the cancellation factor

\begin{tabular}{|c|c|c|c|c|c|c|}
\hline \multirow[t]{2}{*}{ Weighting function } & \multicolumn{3}{|c|}{ Saddle-coil difference spectrum } & \multicolumn{3}{|c|}{ Solenoidal microcoil difference spectrum } \\
\hline & $\begin{array}{l}\text { Cancellation } \\
\mathrm{S} / \mathrm{N}\end{array}$ & $\begin{array}{l}\text { Methanol } \\
\text { S/N }\end{array}$ & $\begin{array}{l}\text { Cancellation } \\
\text { factor }\end{array}$ & $\begin{array}{l}\text { Cancellation } \\
\mathrm{S} / \mathrm{N}\end{array}$ & $\begin{array}{l}\text { Methanol } \\
\text { S/N }\end{array}$ & $\begin{array}{l}\text { Cancellation } \\
\text { factor }\end{array}$ \\
\hline None & 54.12 & 389.4 & 7.2 & 5.27 & 139.7 & 27 \\
\hline Gaussian $(0.5 \mathrm{~Hz})$ & 133.6 & 1086 & 8.1 & 5.53 & 222.1 & 40 \\
\hline Lorentzian (1 Hz) & 136.7 & 1180 & 8.6 & 5.78 & 257.3 & 45 \\
\hline Sinebell $(\mathrm{sbs}=0.3, \mathrm{sbs}=-0.3)$ & 132.6 & 1191 & 9.0 & 5.64 & 311.3 & 55 \\
\hline
\end{tabular}


lation of the Lorentzian peaks is more difficult than the Gaussian peak shape. Weighting functions are typically used to suppress the tails of the NMR peaks, to reduce noise, and to improve resolution. While moderate weighting functions were enough to improve the cancellation factor for the overlapped Gaussian peaks, the tails causing the residual signal for the Lorentzian peaks were too large to suppress significantly.

The saddle-coil difference probe has several advantageous features that differ from the solenoidal microcoil difference probe. The saddle coils themselves detect a larger volume $(25 \mu \mathrm{L})$ than the solenoidal microcoils $(72 \mathrm{~nL})$. With a larger volume, the concentration limit of detection $\left(4 \mathrm{mM}{ }^{1} \mathrm{H}\right.$, one scan) is a factor of 10 better than the microcoil difference probe. The sensitivity of the saddle-coil difference probe could be improved further by utilizing a better filling factor (sample volume/coil volume). Using the 5-mm support tube for the coils and the $3 \mathrm{~mm}$ NMR sample tube $(2.42 \mathrm{~mm}$ i.d.), the filling factor is only about $23 \%$. If the 5-mm coil support tube could be replaced with a device that supports the coil from the outer circumference, a larger sample tube could be inserted inside the coils. This system would improve the filling factor and increase the amount of sample detected. Another difference compared to the microcoil probe is that the saddle coils allow a sample or a dual-sample tube to be inserted into the probe using the spectrometer's upper barrel and turbine. Even more importantly, the sample can be spun to reduce the effects of equatorial static magnetic field inhomogeneities. The saddle-coil difference probe is also equipped with a pair of actively shielded, pulsed field gradient coils that can produce an axial magnetic field gradient. The gradient is used in gradient shimming protocols for automated axial shimming and can be used in experiments such those involving diffusion.

\section{Protein-ligand binding study}

One promising application of the saddle-coil difference probe is in the area of bioanalytical NMR. In particular, protein-ligand studies are important for drug development activities. A CPMG experiment was used to demonstrate how a difference experiment traditionally performed on two separate samples could be performed with the saddle-coil difference probe in one step. This relaxationedited experiment is used to reveal ligand-protein interactions by comparing a reference sample of ligands to a sample of the ligands in the presence of the target protein [8]. If a ligand binds to the protein, the effective transverse relaxation rate is increased; otherwise, the ligand relaxation rate is not affected. Figure 7 shows the effective $T_{2}$ decay curves for GSH and serine with and without GST and the decay curve of GST itself. The curves were fit with an exponential curve to determine the effective $T_{2}$ time for each component. The protein displays a typically short effective $T_{2}$ value of $0.04 \mathrm{~s}$ while the free GSH and serine have values of 0.87 and $1.00 \mathrm{~s}$, respectively. However, in the presence of GST, GSH shows a suppressed re-

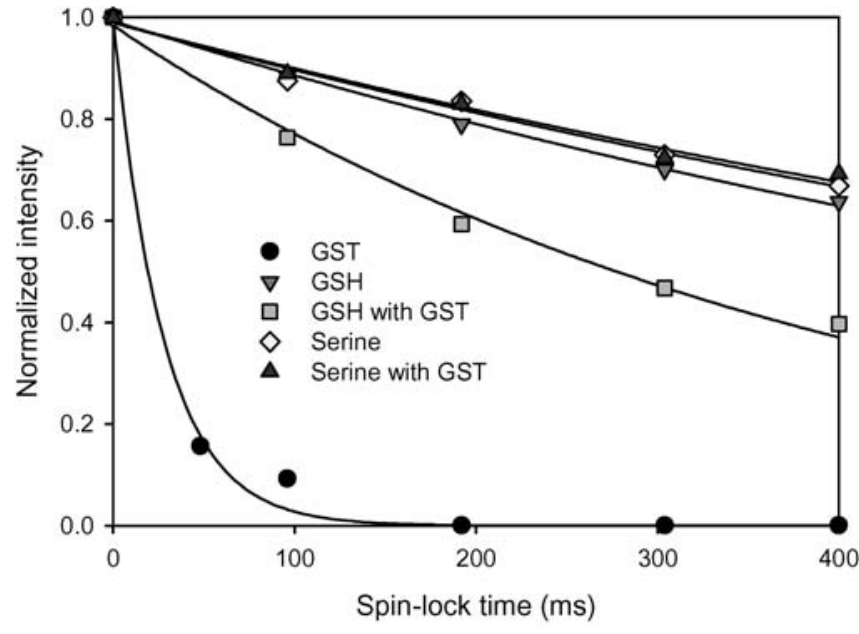

Fig. 7 Normalized intensity decay curves of signals from GST (black circles, $0.80 \mathrm{ppm}$ ), and GSH (red triangle, green square, $3.74 \mathrm{ppm}$ ) and serine (yellow diamond, blue triangle, $3.94 \mathrm{ppm}$ ) in the presence and absence of the protein, respectively. The data were fit to exponential decay curves to derive the effective $T_{2}$ values

laxation time of $0.41 \mathrm{~s}$ while serine is about the same at $1.03 \mathrm{~s}$, indicating that the GSH is binding to the protein. A spin-lock time of $400 \mathrm{~ms}$ was chosen to ensure that the protein signal would not interfere with the difference experiment and to provide a large difference between the free and bound ligand signal intensities. Improving the sensitivity of the difference probe would allow us to access lower ligand:protein ratios, which would magnify the changes in apparent $T_{2}$ values and allow a shorter spinlock time to be used.

Figure 8a shows a spectrum of the ligand sample after a spin-lock time of $400 \mathrm{~ms}$. Some of the GSH peaks are affected by J-coupling modulation [16], but the peak at $3.74 \mathrm{ppm}$ is a singlet and can be used in this experiment. The J-coupling for serine is small enough that both peaks can be used. Figure $8 \mathrm{~b}$ shows a spectrum taken of the ligand-protein mixture with the same spin-lock time. Notice that the GSH (3.74 ppm) is attenuated while the serine signals remain the same, indicating binding of GSH to GST. Traditionally, spectra (a) and (b) would be transferred to a software program where the spectra would be subtracted either manually or using an automated program, which is unnecessary using the difference probe. In Fig. 8c, the CPMG experiment with a spin-lock time of $400 \mathrm{~ms}$ was used to produce a difference spectrum of the two samples with the saddle-coil difference probe with both samples inside the probe at the same time. The serine peaks in Fig. 8c show the characteristic dispersion pattern of subtracted peaks while the GSH (3.74 ppm) is clearly visible, indicating binding to the protein. The linewidth in Fig. $8 \mathrm{c}$ for GSH is $5.1 \mathrm{~Hz}$, which is broad for typical spectra obtained with the difference probe. One reason for the broad lines is the fact that there is no lock circuit to compensate for drift during this relatively long experiment. In addition, the sample was not spun during the CPMG experiment, so transverse field inhomogeneities were not suppressed. 


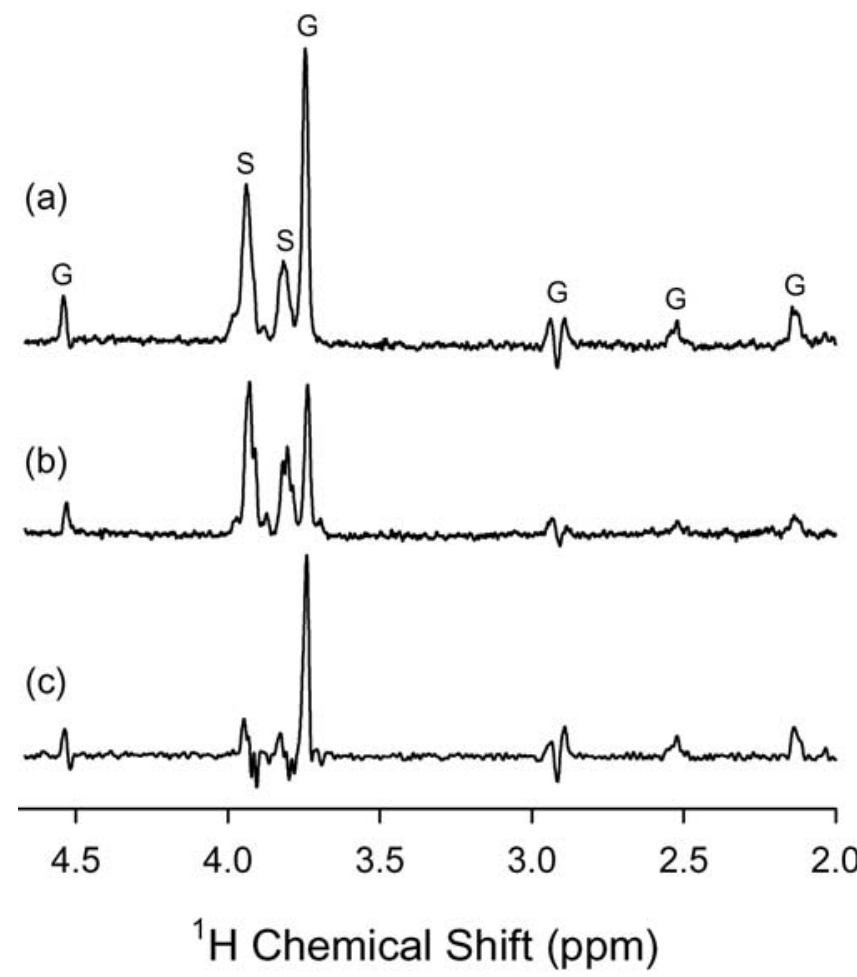

Fig. 8 a Spectra of the ligand sample (10 mM GSH $(G)$ and $10 \mathrm{mM}$ serine $(S)$ ) and b the protein-ligand mixture (10 mM GSH, $10 \mathrm{mM}$ serine, and $6.25 \mathrm{~g} / \mathrm{L} \mathrm{GST}$ ) were taken with the saddle-coil difference probe separately to show the individual spectra, and c together simultaneously to show the difference spectrum. The serine peaks show the dispersion character of cancelled peaks while the GSH peak $(3.74 \mathrm{ppm})$ is clearly visible, indicating a change in its effective $T_{2}$ relaxation due to binding with GST. Note: the other GSH peaks are also visible, but peaks at 4.6 and $2.8 \mathrm{ppm}$ exhibit J-coupling modulation

We also explored the use of composite pulses in combination with the CPMG pulse sequence. Usually, composite pulses are used to compensate for poor magnetic field homogeneity produced by the excitation coil, i.e., the $\mathrm{B}_{1}$ field homogeneity. While the field homogeneity of the saddle coils was good, with the top coil at $81 \%$ (measured as the $\mathrm{S} / \mathrm{N}$ ratio of the $450^{\circ}$ pulse $/ 90^{\circ}$ pulse) and the bottom coil at $77 \%$, the $90^{\circ}$ pulse times were different for each coil. The top coil had a $90^{\circ}$ pulse time of $26 \mu$ s and the bottom had a $90^{\circ}$ pulse time of $32 \mu$ s at the same transmitter power. While the different $90^{\circ}$ pulse times of the two coils provide an opportunity to compensate for sample differences and match the relative signal intensities of each coil, as one would when optimizing a manual, software subtraction, having disparate $90^{\circ}$ times is not the ideal situation for multipulse experiments. The difference in coil efficiency is due to the fact that the coils are hand-formed and imperfect in shape. The best solution would be to use commercially manufactured coils or improve the handmade process, but a short-term solution was to use composite pulses. An intermediate $90^{\circ}$ and $180^{\circ}$ pulse time was chosen to make the composite pulses for the CPMG experiment. The signal intensity and even the J-coupling modulation were improved by using the composite pulses.

\section{Conclusion}

A new difference probe was developed with saddle-shaped coils that allow for vertically aligned, spinning samples. A $3 \mathrm{~mm}$ NMR tube was modified by using Ultem magnetic susceptibility matched plastic to separate two samples within the tube. The dual-saddle difference probe has a resonant circuit that uses crossed-diode switches to create a phase difference between the two samples' signals, thus creating a difference spectrum automatically through the hardware. The advantage of the difference probe is that two samples can be analyzed simultaneously, thus decreasing the overall analysis time compared to a probe with the same sensitivity. The probe also provides a means of optimizing the difference spectrum on-site before the final acquisition. Because the difference between the signals is completed in the hardware of the probe circuit before reaching the receiver of the spectrometer, the effective dynamic range of the probe is increased as large signals can be suppressed prior to acquisition. One disadvantage is that the signal frequency produced by each coil must be matched using the shims while the postprocessing technique shifts the axes before subtraction.

As a prototype, the performance of the saddle-coil difference probe is currently limited by the construction of the saddle coils. The imperfections in the coils contribute to the mismatched coil efficiencies and to the difficulties of shimming both coils simultaneously. The cancellation factor for the difference probe is approximately 9 with half-height linewidths of 2.1 and $2.4 \mathrm{~Hz}$ for the top and bottom coil, respectively. Based on the best half-height linewidths of the coils shimmed individually, we believe that the simultaneously shimmed linewidths can be improved with more symmetrical and matched coils. Improving the linewidths will improve the sensitivity of the probe as well as the cancellation factor. The current sensitivity of the probe does not compare well with commercially built probes; however, the filling factor and the circuit efficiency can be improved, and the probe could be tuned for a higher field magnet to improve its performance. To demonstrate the practical use of the saddle-coil difference probe, a CPMG-based relaxation-edited difference experiment was performed using a binding ligand, GSH, and a nonbinding ligand, serine, with the enzyme GST. The relaxation-edited difference experiment was performed once on both samples simultaneously to produce a single difference spectrum. As the first double, saddlecoil NMR probe capable of hardware-based subtraction of two samples, the saddle-coil difference probe represents a new approach to difference spectroscopy for NMR.

Acknowledgements We would like to express our appreciation to Randall Replogle for his precision machine work and to Tim Selby for construction of the crossed diodes. Megan Macnaughtan thanks Pfizer, Inc. and Purdue Research Foundation for graduate fellowships. This project has been funded in part by the NSF (CHE 95-31693) and the Alfred P. Sloan Foundation. 


\section{References}

1. Skoog DA, Holler FJ, Nieman TA (1998) Principles of instrumental analysis. Saunders, Philadelphia and Hartcourt Brace, Orlando

2. Macnaughtan MA, Hou T, MacNamara E, Santini RE, Raftery D (2002) J Magn Reson 156:97-103

3. Shuker SB, Hajduk PJ, Meadows RP, Fesik SW (1996) Science 274:1531-1534

4. Medek A, Hajduk PJ, Mack J, Fesik SW (2000) J Am Chem Soc 122:1241-1242

5. Meyer B, Peters T (2003) Angew Chem Int Ed 42:864-890

6. Chen A, Shapiro MJ (2000) J Am Chem Soc 122:414-415

7. Mayer M, Meyer B (1999) Angew Chem Int Ed 38:1784-1788
8. Hajduk PJ, Olejniczak ET, Fesik SW (1997) J Am Chem Soc 119:12257-12261

9. Carr HY, Purcell EM (1954) Phys Rev 94:630-638

10. Meiboom S, Gill D (1958) Rev Sci Instrum 29:688-691

11. DeRidder BP, Dixon DP, Beussman DJ, Edwards R, Goldsbrough PB (2002) Plant Physiol 130:1497-1505

12. Sheehan D, Meade G, Foley VM, Dowd CA (2001) Biochem J 360:1-16

13. Hou T, Smith J, MacNamara E, Macnaughtan M, Raftery D (2001) Anal Chem 73:2541-2546

14. Levitt MH, Freeman R (1981) J Magn Reson 43:65-80

15. Doty FD, Entzminger G, Yang A (1998) Concepts Magn Reson 10:133-156

16. Derome, AE (1997) Modern NMR techniques for chemistry research, vol 6. Pergamon, New York 